\title{
Recoverable sets of initial conditions and their use for aircraft flight planning after a loss of control event
}

\author{
Kevin McDonough* Ilya Kolmanovsky ${ }^{\dagger}$ Ella Atkins ${ }^{\ddagger}$ \\ The University of Michigan, Ann Arbor, MI, 48109, USA
}

\begin{abstract}
This paper addresses the computation and prediction of recoverable sets for aircraft flight control, especially after a loss of control event. A recoverable set is the set of all initial conditions for which there exists a control sequence such that the ensuing system trajectories satisfy the imposed constraints while the aircraft is stabilized to a specified trim point. In our approach, the recoverable sets are approximated as projections of safe sets for the closed-loop linearized system dynamics extended with the additional states of an auxiliary system that generates the recovery sequence. We illustrate the computation of the recoverable sets and how these sets evolve with changes in flight conditions. A reshaping transformation is proposed to estimate a subset of the recoverable set at one flight condition given knowledge of the recoverable set at another given flight condition. The reshaping transformations of the recoverable sets that include scalar and vector scalings of their vertex representation are studied in more detail. Approaches to checking state recoverability based on nonlinear models are also discussed.
\end{abstract}

\section{Nomenclature}

$O_{\infty} \quad$ A safe set

$R_{\infty}$ A recoverable set

$S \quad$ wing surface area

$b$ wing span

$c \quad$ mean wing chord

$\rho \quad$ air density

$U_{0} \quad$ nominal air speed

$\Delta x \quad$ A perturbed state in a linearized system model

$u \quad \mathrm{X}$ component of aircraft airspeed

$\alpha \quad$ angle of attack

$q$ pitch rate

$\theta \quad$ pitch angle

$\beta \quad$ sideslip angle

$p$ roll rate

$r$ yaw rate

$\phi \quad$ roll angle

$\delta_{e} \quad$ elevator angle

$\delta_{t h} \quad$ throttle position

$\delta_{r} \quad$ rudder angle

$\delta_{a} \quad$ aileron angle

$\Re \quad$ set of real numbers

\footnotetext{
${ }^{*}$ Graduate Student Researcher, Department of Aerospace Engineering

$\dagger$ Professor, Department of Aerospace Engineering

$\ddagger$ Associate Professor, Department of Aerospace Engineering
} 


\section{Introduction}

Loss of control is the most common cause of commercial and general aviation accidents. ${ }^{1-4,11-15,19-21,25,26}$ Loss of control (LOC) results when an aircraft exits its safe operating envelope, a condition which can be anticipated by modeling the envelope and monitoring the aircraft's progress through this envelope over time. This paper explores a control theoretic approach to real-time envelope estimation in support of flight planning and LOC avoidance. Specifically, once model parameters are estimated through the on-board system identification algorithm for a given flight condition, this paper defines a method of identifying safe sets of trim points within the envelope.

In this paper, we consider the use of recoverable sets as means of determining whether or not safe transitions to specified trim points can be executed. Recoverable sets are sets of initial conditions of the closed-loop system for which there exists a control sequence such that when this sequence is added to the nominal controller, the ensuing trajectories satisfy state and control constraints.

Assuming the aircraft remains under closed-loop control after a LOC event and on-board system identification is used to estimate a linearized model of the closed-loop system dynamics at one flight condition, we use the identified model and a scaling transformation as a basis for predicting the recoverable sets at other flight conditions. To facilitate the on-board computations, the recovery sequence is generated as an output of a low-dimensional auxiliary system, and the recoverable set computation reduces to computing a safe set for the extended system dynamics.

Other works in this area, see e.g. ${ }^{1-4,10-15,17,18}$ have focused on exploiting the continuous-time nonlinear models but, to the author's knowledge, have not demonstrated the feasibility of on-board computations. Besides simplifying the on-board computations, our reliance on the discrete-time linearized models is motivated by the consideration that in a LOC scenario it is unlikely that large alterations in the aircraft's flight conditions will be attempted. Instead it is more likely that a progression of several small changes/transitions through trim state space will be executed. Furthermore, if the constraints are affine and the system model is linear discrete-time, the recoverable set computed with our approach is polyhedral and is easily storable and manipulable.

To further simplify the on-board computations and enable fast prediction of subsets of recoverable sets, in this paper we propose the use of a scaling transformation constructed off-board and applied on-board. This transformation can be applied to a recoverable set estimated at one flight condition to estimate a subset of a recoverable set at another flight condition. The recoverable sets estimated at different flight conditions are then used by a flight planning algorithm.

We will also consider the computation of recoverable sets based on a nonlinear model. Our method uses precomputed sensitivity matrices and an established set of control sequences to speed up the computation.

The paper is organized as follows. In Section II, we discuss the aircraft models used in this work. In Section III we provide formal definitions of safe and recoverable sets and our approach to the computation of the recoverable sets based on the augmented system dynamics. In Section IV, we examine and comment on recoverable set dependence on flight conditions as inferred from our models. In Section V we consider an approximation technique to adjust the boundary of the recoverable set if model parameters change in a small range. In Section VI, we consider the development of a scaling transformation to link subsets of recoverable sets computed at different flight conditions. In Section VII we demonstrate the presented methods on an aircraft icing example. In Section VIII, we introduce the idea of nonlinear recoverability.

\section{Aircraft Model}

\section{A. Aircraft modeling}

The aircraft model being used in the subsequent developments is NASA's Generic Transport Model (GTM). The GTM is designed to be representative of a typical transport aircraft such as the Boeing 757 or Airbus A320. Data were provided for the GTM in the form of aircraft specifications and look up tables of the aircraft's stability derivatives.

In the GTM, the stability derivatives were obtained using the VorView software package ${ }^{16}$ at a nominal speed of $515.55 \mathrm{ft} / \mathrm{s}$ while Mach number and angle of attack were varied as $M=0.1,0.2, \ldots, 0.8$ and $\alpha=$ $-2,0, \ldots, 12 \mathrm{deg}$. Linear interpolation is used to evaluate stability derivatives between these points. While the stability derivatives were developed using a specific airspeed and a set of altitude values, it is assumed that these stability derivatives apply to other combinations of altitude and airspeed resulting in the same 
Mach number.

Using the stability derivatives and equations found in Appendix A, linearized longitudinal and lateral models are calculated for a given flight condition. The states for the longitudinal model are $x_{\text {long }}=$ $[\Delta u, \Delta \alpha, \Delta q, \Delta \theta]^{T}$. The states for the lateral model are $x_{l a t}=[\Delta \beta, \Delta p, \Delta r, \Delta \phi]^{T}$. As an example, the longitudinal and lateral equations for $U_{0}=515.55 \mathrm{ft} / \mathrm{s}, h=1000 \mathrm{ft}$, and $\alpha=5 \mathrm{deg}$ are,

$$
\dot{x}_{\text {long }}=\left[\begin{array}{cccc}
-0.0102 & -9.7264 & -1.4988 & -32.2 \\
-0.0004 & -0.9992 & 0.9617 & 0 \\
0.0016 & -2.4752 & -0.7972 & 0 \\
0 & 0 & 1 & 0
\end{array}\right]\left[\begin{array}{c}
\Delta u \\
\Delta \alpha \\
\Delta q \\
\Delta \theta
\end{array}\right]+\left[\begin{array}{cc}
4.4286 & 3.33 \\
-0.0856 & 0 \\
-3.3372 & 0 \\
0 & 0
\end{array}\right]\left[\begin{array}{c}
\delta_{e} \\
\delta_{t h}
\end{array}\right],
$$

and

$$
\dot{x}_{l a t}=\left[\begin{array}{cccc}
0.1646 & 0.0033 & -1.0018 & 0.0625 \\
-10.5393 & -3.0745 & 0.5421 & 0 \\
2.7760 & 0.0174 & -0.6265 & 0 \\
0 & 1 & 0 & 0
\end{array}\right]\left[\begin{array}{c}
\Delta \beta \\
\Delta p \\
\Delta r \\
\Delta \phi
\end{array}\right]+\left[\begin{array}{cc}
-0.0059 & -0.0728 \\
7.3283 & 1.6432 \\
0.0226 & -2.2790 \\
0 & 0
\end{array}\right]\left[\begin{array}{c}
\delta_{r} \\
\delta_{a}
\end{array}\right] .
$$

\section{B. Controller modeling}

For the work presented here, once a linear model is found, it is transformed into discrete time using a sampling period of $0.2 \mathrm{sec}$. A stabilizing controller is considered which is designed using discrete time linear quadratic regulator (DLQR) theory. The weighting matrices used in the DLQR problem are diagonal, and chosen as $Q_{\text {long }}=\operatorname{diag}\{0.001,0,0,10\}$ and $R_{\text {long }}=I_{2 \times 2}$ for the longitudinal system and $Q_{\text {lat }}=\operatorname{diag}\{1,0,0,1\}$ and $R_{\text {lat }}=I_{2 \times 2}$ for the lateral system. The same weighting matrices are applied across all flight conditions, and we assume that the controller is regenerated on-board using these weighting matrices if the model changes and assures closed-loop stability.

\section{Safe and Recoverable Sets}

Our computational treatment of recoverable sets presented below is based on procedures to compute safe sets.

\section{A. Safe Sets}

In this work, a safe set is the set of all initial conditions such that the ensuing trajectories of the system do not violate imposed constraints. Consider the following model of closed-loop aircraft dynamics with the nominal controller,

$$
\begin{aligned}
x(k+1) & =A x(k)+B v(k), \\
y(k) & =C x(k)+D v(k),
\end{aligned}
$$

where $x$ is the $n$-vector state, $y$ is the $m$-vector output, and $v$ is a $p$-vector adjustable input that additively augments the nominal controller output. Since (3) represents a model of closed-loop system, A is assumed to be Schur-that is, all eigenvalues of the closed-loop discrete-time system have magnitudes less than 1.

The output $y$ is subject to pointwise-in-time output constraints specified as requirements

$$
y(k) \in Y, \forall k \geq 0,
$$

where $Y$ is a given set. The constraints (4) can reflect state variable limits, and since (3) is a model of the closed-loop system, (4) can also reflect actuator magnitude or rate limits as these can be restated as constraints on state variables of the closed-loop system. Constraints can also be imposed to ensure that the aircraft operates in a range where model (3) provides an adequate representation of system dynamics. The constraints are assumed to be affine and $Y$ is defined as

$$
Y=\{y: G y \leq F\} .
$$

In (5), $G \in \Re^{r \times n}, F \in \Re^{r}$ and $r$ is the number of constraints imposed on the output. 
Using the above formulation, a safe set, $O_{\infty}$, is defined for (3)-(5) with $v(k) \equiv 0$ as

$$
O_{\infty}=\left\{x(0) \in \Re^{n}: y(k)=C A^{k} x(0) \in Y, \forall k \geq 0\right\} .
$$

If $A$ is Schur, $(C, A)$ is observable, $0 \in \operatorname{int} Y$ and $Y$ is compact, thus $O_{\infty}$ in (6) is positively-invariant, finitely-determined and is a polytope (bounded polyhedron). ${ }^{9}$ Finite determination means that there exists $t^{*}$ such that $O_{\infty}=O_{t}$ for all $t \geq t^{*}$, where

$$
O_{t}=\left\{x(0) \in \Re^{n}: y(k)=C A^{k} x(0) \in Y, \text { for } k=0, \cdots, t\right\},
$$

or, equivalently,

$$
O_{\infty}=O_{t^{*}}=\left\{x(0) \in \Re^{n}: G C A^{k} x(0) \leq F, \text { for } k=0, \cdots, t^{*}\right\} .
$$

In practice, $t^{*}$ is usually comparable to the settling time of the closed-loop system and not all the constraints forming $O_{\infty}$ in (8) are necessary as many are redundant or almost redundant and can be eliminated. The advantage of eliminating the redundant/almost redundant constraints is that the storage memory requirements are reduced and subsequent computations involving $O_{\infty}$ are simplified. For the generalizations of the safe sets to systems with additive set-bounded disturbances, see reference ${ }^{35}$ and references therein and for systems with polytopic uncertainty, see reference ${ }^{28}$ and references therein.

\section{B. Recoverable sets}

The viability kernel, $V_{\infty}$ for (3)-(5) is defined as the set of all initial conditions $x(0)$ for which there exists a sequence $v(\cdot)$ that enforces the constraints, i.e.,

$$
V_{\infty}=\{x(0): \exists v(\cdot) \text { such that } y(k) \in Y \forall k \geq 0\} .
$$

Clearly, $O_{\infty} \subset V_{\infty}$, the sequence $v(\cdot)$ is specific to each initial condition, $x(0)$, and is referred to as the recovery sequence. With the addition of the recover sequence, $V_{\infty}$ can be much larger than $O_{\infty}$.

In our approach to developing an inner approximation to $V_{\infty}$, we assume that the recovery sequence is generated by an auxiliary system of the form,

$$
\begin{aligned}
& v(k)=\bar{c}+\bar{C} \bar{x}(k), \\
& \bar{x}(k+1)=\bar{A} \bar{x}(k),
\end{aligned}
$$

where $\bar{A}$ is a Schur matrix and $\bar{x}$ is the $\bar{n}$-vector state of the auxiliary system (8). We denote the safe set for (3)-(5), (10) as $O_{\infty}^{e x t}$,

$$
O_{\infty}^{e x t}=\left\{(\bar{c}, \bar{x}(0), x(0)) \in \Re^{p+\bar{n}+n}: y(k) \in Y, \forall k \geq 0\right\} .
$$

The recoverable set is defined as a projection of $O_{\infty}^{e x t}$ on the last $n$ coordinates,

$$
R_{\infty}=\left\{x(0) \in \Re^{n}: \exists \bar{c}, \bar{x}(0) \text { such that }(\bar{c}, \bar{x}(0), x(0)) \in O_{\infty}^{e x t}\right\} .
$$

Note that since the constraints (5) are affine, both $O_{\infty}^{e x t}$ and $R_{\infty}$ are polyhedral. Note also that the recoverable sets (12) are positively-invariant in a sense that the next state of the system generated under the recovery sequence remains recoverable. The introduction of auxiliary system (10) is motivated by reference ${ }^{33}$ where it was used in the formulation of the Extended Command Governor, however, here-and similarly to reference ${ }^{31}$ we apply it to the approximation of recoverable sets.

Given $x(0)$, computing an appropriate $\bar{c}$ and $\bar{x}(0)$ reduces to a problem of the type,

$$
\begin{gathered}
\frac{1}{2} \bar{c}^{\mathrm{T}} \bar{c}+\frac{1}{2} \bar{x}(0)^{\mathrm{T}} P \bar{x}(0) \rightarrow \min , \\
\text { subject to }(\bar{c}, \bar{x}(0), x(0)) \in O_{\infty}^{e x t},
\end{gathered}
$$

where $P=P^{\mathrm{T}}>0$. The minimization in (13) aims at preserving the operation with the nominal controller and avoiding the unnecessary use of recovery sequence. Computationally, (13) is a quadratic programming problem. By using 1 or $\infty$ norms in (13) instead of quadratic penalties, a closely related linear programming formulation can be obtained. Once $\bar{x}(0)$ and $\bar{c}$ are determined, the execution of (10) yields a recovery sequence for a given $x(0)$ that enforces the given constraints and under reasonable assumptions ensures that $x(t) \rightarrow 0$ as $t \rightarrow \infty$. 
Several choices exist for the selection of the auxiliary dynamics (10), including the use of the shift register and of Laguerre sequence generators. In case of the shift register, the recovery sequence becomes equal to $\bar{C}$ after $H+1$ steps and $\bar{A}$ and $\bar{C}$ have the form

$$
\bar{A}=\left[\begin{array}{cccc}
\bar{S}_{1} & 0 & \cdots & 0 \\
0 & \bar{S}_{2} & \cdots & 0 \\
\vdots & \vdots & \ddots & \vdots \\
0 & 0 & \cdots & \bar{S}_{p}
\end{array}\right], \quad \bar{C}=\left[\begin{array}{cccc}
\bar{T}_{1} & 0 & \cdots & 0 \\
0 & \bar{T}_{2} & \cdots & 0 \\
\vdots & \vdots & \ddots & \vdots \\
0 & 0 & \cdots & \bar{T}_{p}
\end{array}\right]
$$

and

$$
\bar{S}_{i}=\left[\begin{array}{ccccc}
0 & 1 & 0 & \cdots & 0 \\
0 & 0 & 1 & \cdots & 0 \\
\vdots & \vdots & \vdots & \ddots & \vdots \\
0 & 0 & \cdots & \cdots & 1 \\
0 & 0 & \cdots & \cdots & 0
\end{array}\right] \in R^{(H+1) \times(H+1)}, \quad \bar{T}_{i}=\left[\begin{array}{ccccc}
1 & 0 & \cdots & \cdots & 0
\end{array}\right] \in R^{1 \times(H+1)}
$$

The Laguerre's sequence generators correspond to the choices,

$$
\bar{S}_{i}=\left[\begin{array}{ccccc}
\alpha & \beta & -\alpha \beta & \alpha^{2} \beta \ldots & \\
0 & \alpha & \beta & -\alpha \beta & \ldots \\
0 & 0 & \alpha & \beta & \ldots \\
0 & 0 & 0 & \alpha & \ldots \\
\vdots & \vdots & \vdots & \vdots & \vdots
\end{array}\right], \quad \bar{T}_{i}=\sqrt{\beta}\left[\begin{array}{lllll}
1 & -\alpha & \alpha^{2} & -\alpha^{3} & \ldots
\end{array}\right],
$$

where $\beta=1-\alpha^{2}, 0 \leq \alpha \leq 1$ are selectable parameters of the auxiliary dynamics. The choice $\alpha=0$ corresponds to the shift register. Numerical examples suggest that low dimensional Laguerre's sequence generators for $\alpha \neq 0$ are capable of producing a rich set of recovery sequences. The use of Laguerre's sequences is motivated by reference ${ }^{33}$ that considered Extended Command Governors, here they are applied to approximations of recoverable sets.

To incorporate the extended dynamics with the nominal closed loop dynamics, note that

$$
u(k)=-K x(k)+v=-K x(k)+\bar{c}+\bar{C} \bar{x}(k),
$$

where $K$ is the feedback gain and thus,

$$
u(k)=\left[\begin{array}{lll}
-K & I & \bar{C}
\end{array}\right]\left[\begin{array}{c}
x(k) \\
\bar{c} \\
\bar{x}(k)
\end{array}\right] .
$$

Thus, when one constructs $O_{\infty}^{e x t}$ with the inclusion of a nominal controller, the state vector becomes $\left[\begin{array}{lll}x(k) & \bar{c} & \bar{x}(k)\end{array}\right]^{T}$ with

$$
A_{\text {ext }}=\left[\begin{array}{ccc}
A & B & B \bar{C} \\
0 & I & 0 \\
0 & 0 & \bar{A}
\end{array}\right], \quad C_{\text {ext }}=\left[\begin{array}{ccc}
C & D & D \bar{C} \\
-K & I & \bar{C}
\end{array}\right]
$$

used in place of the nominal $A$ and $C$. The second row of $C_{e x t}$ is introduced to handle the constraints imposed on the control.

\section{Use of recoverable sets for aircraft flight planning}

The recoverable sets can be used in aircraft flight planning to determine a sequence of trim points that can be safely followed by the aircraft. The condition for connectivity and safe transition between trim points can be expressed as

$$
X_{e q}^{n}-X_{e q}^{n+1} \in R_{\infty}^{n+1},
$$


where $X_{e q}^{n}, X_{e q}^{n+1}$ denote the $n$th and $(n+1)$ st equilibrium states of the nonlinear closed-loop system, respectively, and $R_{\infty}^{n+1}$ denotes the recoverable set corresponding to the linearized closed-loop model at $X_{e q}^{n+1}$. With the controller commands corresponding to the $n$th trim point, $X(t)$ converges to $X_{e q}^{n}$ and (19) ensures that the $(n+1)$ st trim condition can be commanded and, with the help of a recovery sequence, achieved without violating the constraints. Note that the knowledge of the actual recovery sequence is not required at the flight planning stage where a safe sequence of trim points can be determined based on condition (19). Reference ${ }^{30}$ exemplifies this approach based on safe rather than recoverable sets in the case of spacecraft relative motion control while enforcing debris avoidance and thrust limit constraints.

In the application of the recoverable sets for aircraft flight planning, we note that $O_{\infty}^{e x t}, R_{\infty}$ can be replaced by their subsets. These subsets can have simple representations and do not need to be positivelyinvariant, thereby resulting in simplifications in (10). The actual recovery sequence is computed by solving $(10),(13)$ with $x(0)=X(t)-X_{e q}^{n+1}$, where $X(t)$ is the current state, and with $O_{\infty}^{e x t}$ corresponding to $X_{e q}^{n+1}$. The generation of the recovery sequence can be performed by using implicit characterization of $O_{\infty}^{n+1}$ (defined through prediction and constraints) in (10)-(11). A simpler scheme to generate a recovery sequence would be to perform an optimization of $\bar{c}$ and $\bar{x}(0)$ subject to the constraint on the one-step-ahead predicted next state of the system, i.e. $x(t+1 \mid t) \in R_{\infty}^{n+1}$ if $x(t) \in R_{\infty}^{n+1}$ The latter scheme may not give the same recovery sequence as the former, and its properties will be studied in future publications.

\section{Examples of safe and recoverable sets}

Figures 1 and 2 show examples safe and recoverable sets computed based on the linearized model for a flight condition of $U_{0}=515.55 \mathrm{ft} / \mathrm{s}, h=1000 \mathrm{ft}$, and $\alpha=5 \mathrm{deg}$. The sets are presented as planar projections. For brevity, not all planar projections are shown. The models used to calculate these sets are the ones presented above.

Figure 1 shows the projections of the safe set and recoverable sets for the longitudinal dynamics where, for the recoverable sets, $\bar{A}$ and $\bar{C}$ correspond to the shift register implementation in with $H=1,2$, and 3 in (15) is used. The constraints imposed on the longitudinal states are

$$
\begin{gathered}
-50 \leq \Delta u \leq 50, \\
-0.0349 \leq \alpha \leq 0.2094, \\
-0.0873 \leq \Delta q \leq 0.0873, \\
-0.6981 \leq \Delta \theta \leq 0.6981, \\
-0.4363 \leq \delta_{e} \leq 0.4363, \\
-20 \leq \delta_{t h} \leq 20,
\end{gathered}
$$

where $\Delta u$ is in $\frac{f t}{s}, \alpha, \Delta \theta$ and $\delta e$ are in $r a d, \Delta q$ is in $\frac{r a d}{s}$, and $\delta_{t h}$ is in percent of maximum thrust. For this work, maximum thrust is constrained to 20,000 pounds.

Note that the recoverable sets contain the safe set and become progressively larger as $H$ increases. The projection showing the greatest benefit to the use of recovery sequences is $\Delta u-\Delta \theta$ where the recoverable set even for $H=1$ is substantially larger than the safe set.

Figure 2 shows the examples of safe and recoverable sets for the lateral dynamics where, for the recoverable sets, the shift register implementation with $H=1,2$, and 3 in (15) is used. The constraints on the lateral states are

$$
\begin{aligned}
&-0.1745 \leq \beta \leq 0.1745, \\
&-0.0873 \leq \Delta p \leq 0.0873, \\
&-0.0873 \leq \Delta r \leq 0.0873, \\
&-0.3491 \leq \Delta \phi \leq 0.2491, \\
&-0.2618 \leq \delta_{a} \leq 0.2618, \\
&-0.3491 \leq \delta_{r} \leq 0.3491,
\end{aligned}
$$

where $\Delta \beta, \Delta \phi, \delta_{r}$, and $\delta_{a}$ are in $\mathrm{rad}$ and $\Delta p$ and $\Delta r$ is in $\frac{\mathrm{rad}}{\mathrm{s}}$. Note that the recoverable sets contain the safe set and become progressively larger as $H$ increases.

It took a total of $0.58 \mathrm{sec}$ to compute the longitudinal and lateral safe sets, $1.89 \mathrm{sec}$ to compute the $H=1$ recoverable sets, $3.55 \mathrm{sec}$ to compute the $H=2$ recoverable sets, and 6.96 sec to compute the $H=3$ 
recoverable sets. The computations were run on a $2.67 \mathrm{GHz}$ processor laptop with $4 \mathrm{~GB}$ of RAM and 32-bit Matlab 2010a, and used the implementation of the safe set computation based on Pluymers et al. ${ }^{28}$

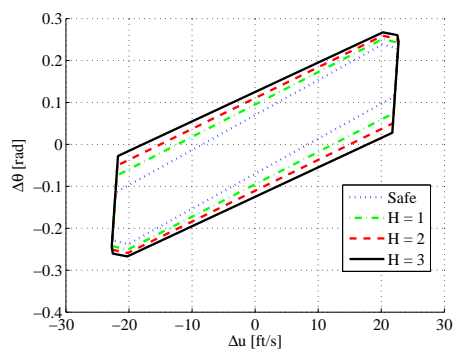

(a) $\Delta u-\Delta \theta$

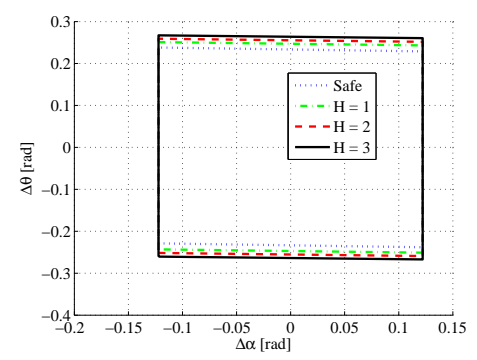

(b) $\Delta \alpha-\Delta \theta$

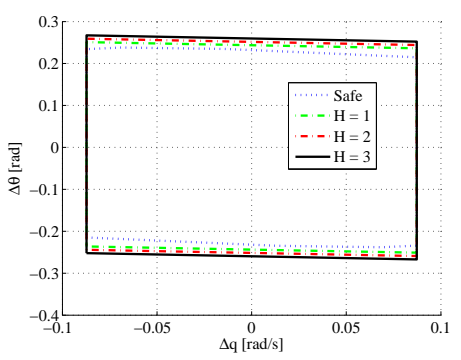

(c) $\Delta q-\Delta \theta$

Figure 1: Projections of longitudinal safe sets, and of recoverable sets for $H=1, H=2$, and $H=3$.

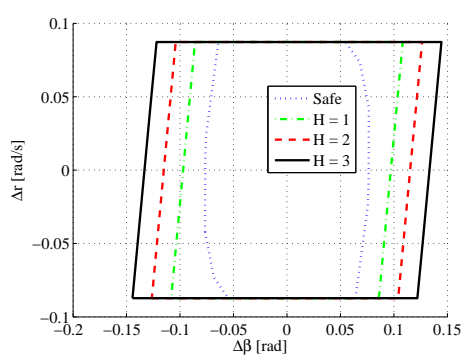

(a) $\Delta \beta-\Delta r$

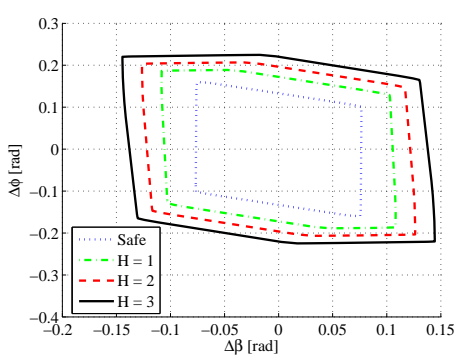

(b) $\Delta \beta-\Delta \phi$

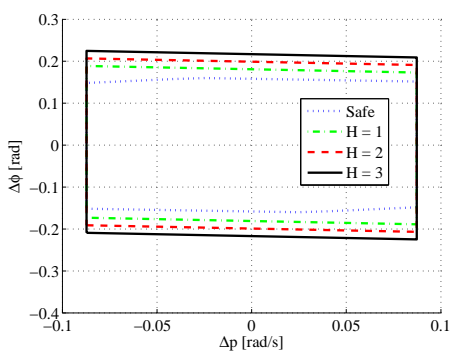

(c) $\Delta r-\Delta \phi$

Figure 2: Projections of lateral safe sets and recoverable sets for $H=1, H=2$, and $H=3$.

Figures 3a-3b demonstrate the difference in recoverable sets for the flight condition $U_{0}=500 \mathrm{ft} / \mathrm{s}$, $h=1000 \mathrm{ft}$, and $\alpha=2 \mathrm{deg}$ when a Laguerre's sequence is used to generate the augmented system instead of the shift register. Note that while the recoverable sets are similar, the recoverable sets generated using the Laguerre's sequence are generally larger. For the computations, $\alpha=0.5$ in (16).

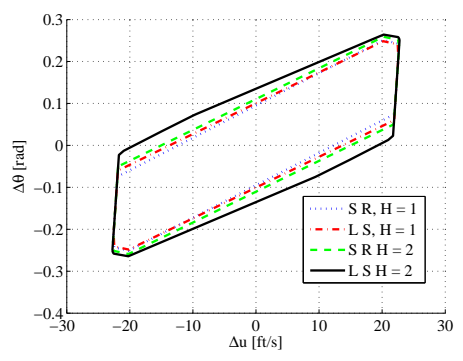

(a) $\Delta u-\Delta \theta$

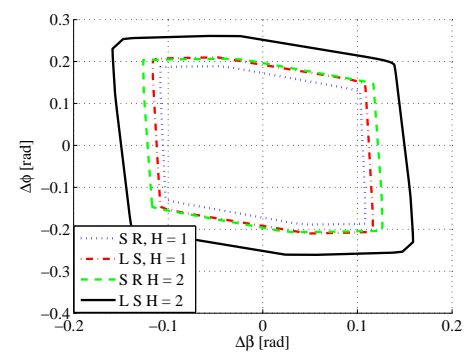

(b) $\Delta \beta-\Delta \phi$

Figure 3: Differences between Laguerre's sequence (LS) and shift register (SR) recoverable sets generated for $H=1$ and $H=2$.

The approach presented above can also handle additive set-bounded disturbances, see reference. ${ }^{35}$ To describe briefly the main ingredients, in the case of additive set-bounded disturbances/uncertainties, (6) becomes

$$
O_{\infty}=\left\{x(0) \in \Re^{n}: y(k)=C A^{k} x(0) \in Y, \forall k \geq 0 \text { and } \forall w \in W\right\},
$$


Table 1: Table of flight conditions used in Figures 5 and 6.

\begin{tabular}{clll}
\hline Flight Condition & $h[f t]$ & $u_{0}\left[\frac{f t}{s}\right]$ & $\alpha_{0}[$ deg $]$ \\
\hline 1 & 1000 & 500 & 4 \\
2 & 1000 & 800 & 4 \\
3 & 20000 & 500 & 4 \\
4 & 20000 & 800 & 4 \\
\hline
\end{tabular}

where

$$
y(k)=C A^{k} x(0)+\sum_{t=0}^{k-1} C A^{(k-t-1)} B w(k)+D w(k),
$$

and $W$ is a bounded set of possible disturbances values. Subsequent definitions, such as (12), are modified similarly. Figures $4 \mathrm{a}$ and $4 \mathrm{~b}$ demonstrate the effects of bounded disturbances for $H=1$ recoverable sets for the flight condition $U_{0}=500 \mathrm{ft} / \mathrm{s}, h=1000 \mathrm{ft}$, and $\alpha=2 \mathrm{deg}$. For Figure 4a, the bounded disturbance occurs in the $\Delta u$ state and for Figure $4 \mathrm{~b}$ the bounded disturbance occurs in the $\Delta p$ state.

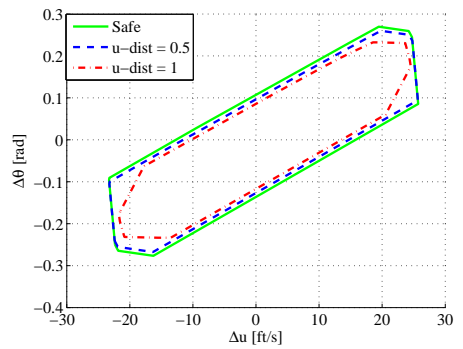

(a) $\Delta u-\Delta \theta$

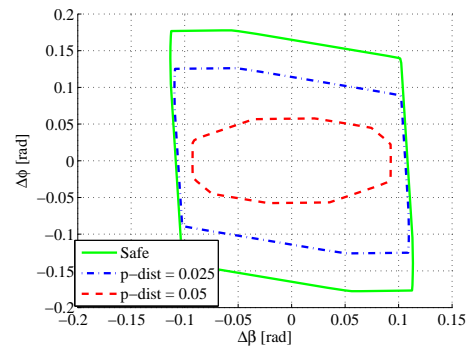

(b) $\Delta \beta-\Delta \phi$

Figure 4: The effects of additive set-bounded disturbances on $H=1$ recoverable sets. Note that as the disturbance increases in size, the recoverable set decreases.

\section{Recoverable Set Dependence on Flight Condition}

The recoverable sets are dependent on the flight condition for which they are being calculated. The dependence on flight condition comes primarily from two sources. The first source is the dependence of the matrix entries on the dynamic pressure, $p_{d}=1 / 2 \rho U_{0}^{2}$, see equations summarized in Appendix $\mathrm{A}$. The second source is in the dependence of the stability derivatives on Mach number and angle of attack.

Figures 5 and 6 demonstrate the effect that different flight conditions have on the recoverable sets. A large difference in altitude, $1000 \mathrm{ft}$ to $20000 \mathrm{ft}$ has less of an effect than a moderate change in speed, $500 \mathrm{ft} / \mathrm{s}$ to $800 \mathrm{ft} / \mathrm{s}$. This is reasonable when considering the dependence of the dynamic pressure on the air density and airspeed: Though $p_{d}$ is dependent on the air density $\rho$, it also depends on the square of the airspeed $U_{0}$. Hence, changes in $U_{0}$ affect the dynamic pressure more than changes in $\rho$. Table 1 summarizes four different flight conditions (FC1, FC2, FC3, FC4) for which the computations of the recoverable sets for the shift register implementation and $H=1$ were performed.

If parameters of the system model change as a result of a LOC event, it is of interest to develop simple computational procedures to predict how safe and recoverable sets will change.

The times to compute recoverable sets based on linearized system models (see Section III) are tractable, suggesting that if the model is reconfigured with estimated parameters, computing the recoverable set onboard at the current flight condition may be feasible. On the other hand, predicting safe and recoverable 


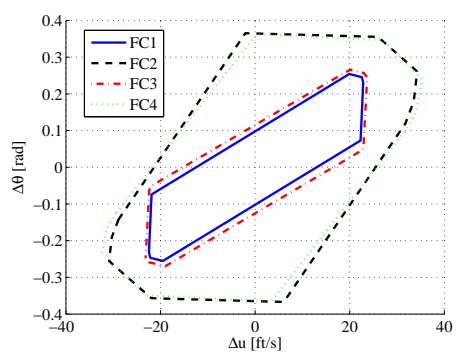

(a) $\Delta u-\Delta \theta$

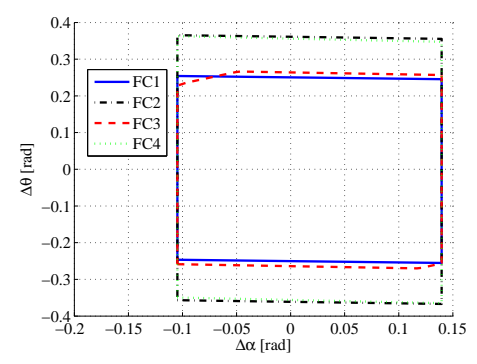

(b) $\Delta \alpha-\Delta \theta$

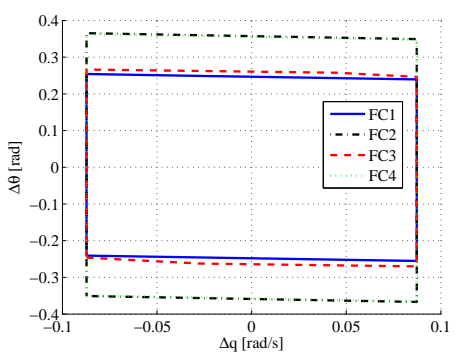

(c) $\Delta q-\Delta \theta$

Figure 5: Projections of longitudinal recoverable sets with $H=1$ at different flight conditions.

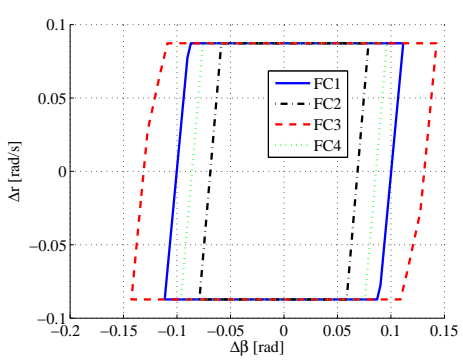

(a) $\Delta \beta-\Delta r$

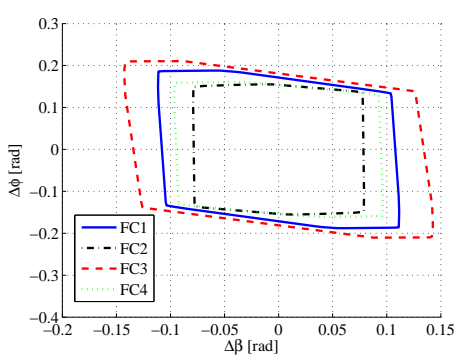

(b) $\Delta \beta-\Delta \phi$

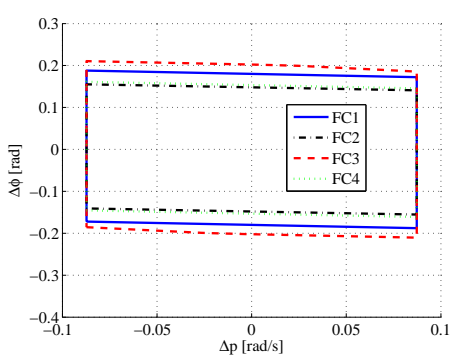

(c) $\Delta p-\Delta \phi$

Figure 6: Projections of lateral recoverable sets for $H=1$ at different flight conditions.

sets at other flight conditions not yet experienced by the aircraft by repeating these computations can add to computation time.

In the next Section, we first consider a simple sensitivity technique that can be used for quick adjustment of the safe/recoverable set representation in response to changes in model parameters. We then describe, in Section VI, an approach based on a scaling transformation that can be applied to safe or recoverable sets computed at one flight condition to predict subsets of safe/recoverable sets at another flight condition. As discussed in Section III, knowledge of the subsets of recoverable sets is sufficient for flight planning.

\section{Sensitivity Technique to Predict Changes in Safe and Recoverable Sets}

Given that the recoverable set computation reduces to the computation of the safe set, $O_{\infty}^{e x t}$ we consider the safe set computations in more detail. The constraints forming the safe set, $O_{\infty}$, are of the form (5), i.e.,

$$
G C A^{k} x(0) \leq F
$$

defined for different values of $k$.

Suppose that the matrix $A$ is a function of an $n_{\theta}$-vector parameter, $\theta$, and suppose that the current estimate, $\hat{\theta}$, of this parameter has deviated from a nominal value, $\theta^{*}$. Then, for small differences between $\hat{\theta}$ and $\theta^{*}$ we can approximate the constraints $(24)$ as

$$
\left(G C A^{k}\left(\theta^{*}\right)+G C\left(k A^{k-1}\left(\theta^{*}\right)\right) \sum_{i=1}^{n_{\theta}} \frac{\partial A}{\partial \theta_{i}}\left(\theta^{*}\right)\left(\hat{\theta}_{i}-\theta_{i}^{*}\right)\right) x(0) \leq F .
$$

The expression (25) indicates that the bounds of the inequalities defining $O_{\infty}$ can be simply tightened or relaxed to accommodate the effect of parameter changes.

The approach based on (25) is applied only to the non-redundant constraints in the representation of $O_{\infty}$ for $\theta=\theta^{*}$. In principle, large deviations of $\hat{\theta}$ from $\theta^{*}$ can lead to a change in redundancy status of 
the constraints, which is not accounted for by this technique. This approach, with minor modifications, is applicable to computing recoverable sets.

Figures 7a-7c illustrate the effectiveness of using this predictive method. In these examples, $\theta$ is the vector of stability derivatives $C_{L 0}, C_{L u}, C_{L \alpha}, C_{L q}, C_{D 0}, C_{D u}, C_{D \alpha}, C_{D q}$. In Figures 7a-7c, all of the stability derivatives associated with lift are decreased by the given percent and all of the stability derivatives associated with drag are increased by the given percent. Note that for small percent changes, the predicted recoverable set is fairly close to the actual set, but this changes quickly as the percent difference increases. These figures are for the $H=1$ recoverable set at the flight condition $U_{0}=500 \mathrm{ft} / \mathrm{s}, h=1000 \mathrm{ft}$, and $\alpha=2 \mathrm{deg}$.

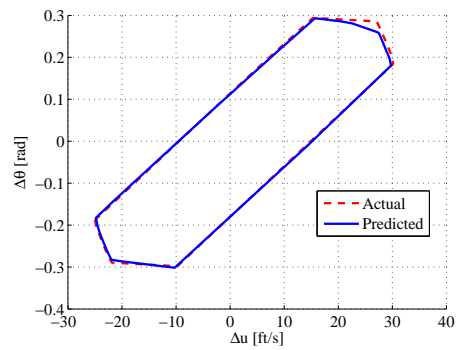

(a) $\Delta u-\Delta \theta, 2 \%$ Change

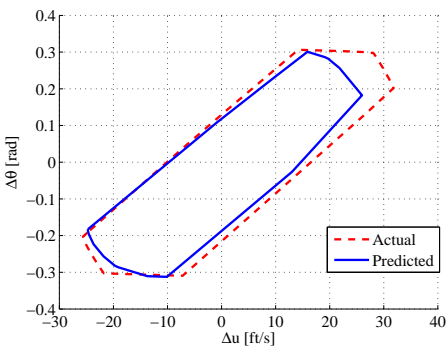

(b) $\Delta u-\Delta \theta, 10 \%$ Change

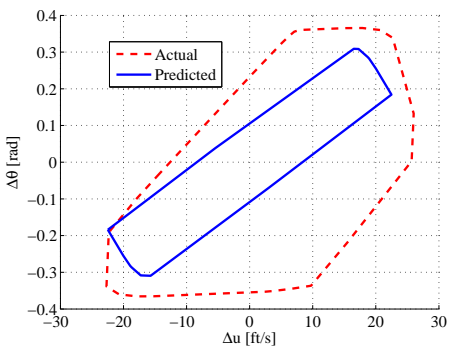

(c) $\Delta u-\Delta \theta, 50 \%$ Change

Figure 7: Examples of the accuracy of the sensitivity technique. Note for large differences accuracy is poor

\section{Scaling Between Recoverable Sets at different Flight Conditions}

The scaling transformation is applied to a recoverable set at one flight condition to guarantee that the scaled set is a subset (not necessarily positively-invariant) of the recoverable set at another flight condition. The scaling transformation is developed conservatively off-line and stored for on-board use so that it can be applied irrespective of degradation or damage conditions ${ }^{\mathrm{a}}$. The use of scaling transformation avoids the need to compute the recoverable sets on-board from scratch, which may be a computationally intensive task.

In the following, we consider two polyhedral recoverable sets, each computed for a different flight condition. The first is described by the set of linear inequalities

$$
G_{1} y \leq F_{1},
$$

where $G_{1} \in \Re^{m \times n}$, and $F_{1} \in \Re^{m}$ and has a set of $n_{1}$ vertices organized into an $n \times n_{1}$ matrix, $V_{1}$. The second is described by the set of linear inequalities

$$
G_{2} y \leq F_{2},
$$

where $G_{2} \in \Re^{k \times n}$, and $F_{2} \in \Re^{k}$ and has a set of $n_{2}$ vertices organized into an $n \times n_{2}$ matrix, $V_{2}$. We assume that the origin is in the interior of both (26) and (27).

Two methods of scaling are now discussed. The first method uses a scalar scaling, while the second uses a vector scaling.

\section{A. Scalar Scaling}

For the scalar scaling, a scalar, $\lambda>0$, is applied to the polytope (26). The scalar scaling transformation is expressed as

$$
T_{\text {scalar }}: x \rightarrow \lambda x \text {. }
$$

To ensure that the scaled polytope is inside (27) it is necessary and sufficient to ensure that the vertices of (26) are mapped inside (27), i.e.,

$$
G_{2} \lambda V_{1} \leq F_{2} .
$$

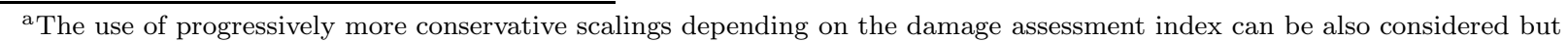
is beyond the scope of the present paper. 
The optimal scaling factor, $\lambda^{*}$, is the largest value of $\lambda$ for which (28) is satisfied and can be computed as

$$
\lambda^{*}=\min _{i, j}\left[\max \left[\frac{F_{2 j}}{G_{2 j} V_{1 i}}, 0\right]\right]
$$

In (29), $G_{2 j}$ denotes the $j$ th row of $G_{2}, V_{1 i}$ denotes the $i$ th column of $V_{1}$ and $F_{2 j}$ denotes the $j$ element of $F_{2}$.

\section{B. Vector Scaling}

For the vector scaling transformation, scalars, $\lambda_{1}>0, \cdots, \lambda_{n}>0$, scale individually each coordinate so that as

$$
T_{\text {vector }}: x=\left(x_{1}, \cdots, x_{n}\right) \rightarrow\left(\lambda_{1} x_{1}, \cdots, \lambda_{n} x_{n}\right) .
$$

Note that

$$
T_{\text {vector }}=\sum_{k=1}^{n} \lambda_{k} T_{k}
$$

where $T_{k}$ is a diagonal matrix with $(k, k)$ element equal to 1 and all other elements being zero.

To ensure that the polytope (26) after the transformation (30) is inside (27) it is necessary and sufficient to ensure that the vertices of (26) are mapped inside (27), i.e.,

$$
\sum_{k=1}^{n} \lambda_{k}\left(G_{2} T_{k} V_{1}\right) \leq F_{2}
$$

The optimal vector transformation is determined by maximizing

$$
J=\sum_{k=1}^{n} \lambda_{k} \rightarrow \max ,
$$

subject to the constraints

$$
\lambda_{k} \geq 0, k=1, \cdots, n,
$$

and subject to the constraint (31). The problem (31)-(33) is a linear programming problem.

\section{More General Transformations}

More general transformations can be considered in the form similar to (30), where

$$
T=\sum_{k=1}^{n_{t}} \lambda_{k} T_{k}
$$

and $T_{k}$ are given matrices.

\section{Numerical results}

Figures 8 and 9 show the results of computing the scaling transformations between recoverable sets for two different flight conditions. The first condition, labelled as 1 in Table 2 , is $U_{0}=500 \mathrm{ft} / \mathrm{s}, h=1000 \mathrm{ft}$, and $\alpha=2 \mathrm{deg}$ and the second flight condition, labelled as 2 in Table 2 , is $U_{0}=580 \mathrm{ft} / \mathrm{s}, h=1000 \mathrm{ft}$, and $\alpha=2$ deg.

For the longitudinal dynamics, the optimal scalar scaling factor is small and produces estimated recoverable sets that are conservative. This is assumed to be due to the auxiliary states introduced to in the recoverable set formulation and their interaction with constraints. The phenomenon, however, is not observed in the lateral dynamics. 


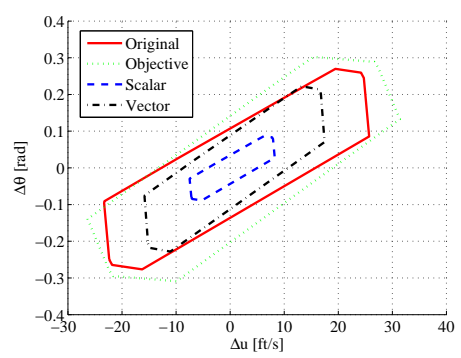

(a) $\Delta u-\Delta \theta$

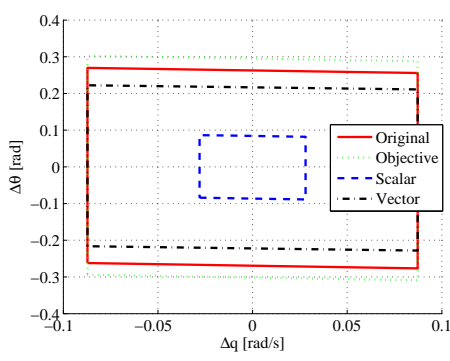

(b) $\Delta \alpha-\Delta \theta$

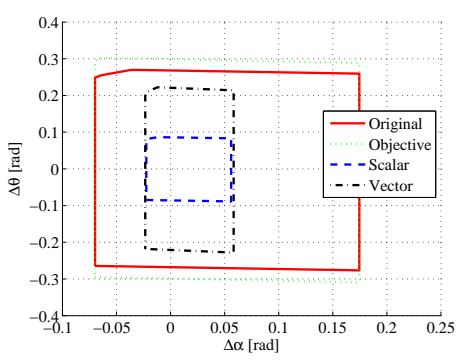

(c) $\Delta q-\Delta \theta$

Figure 8: Example of longitudinal $H=1$ recoverable sets scaled from an original recoverable set to an objective recoverable set. Note that scalar scaling in this case is conservative where as the vector scaling is significantly better.

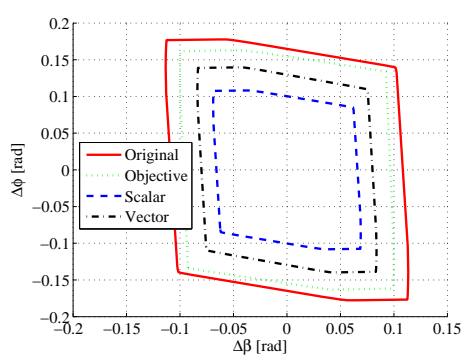

(a) $\Delta \beta-\Delta p$

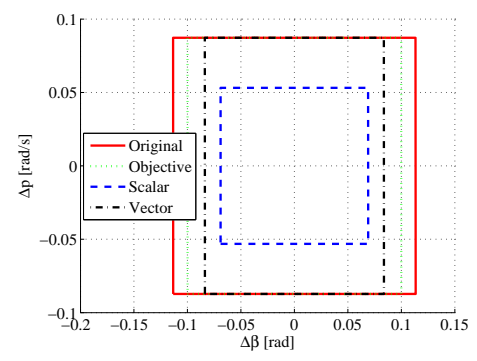

(b) $\Delta \beta-\Delta \phi$

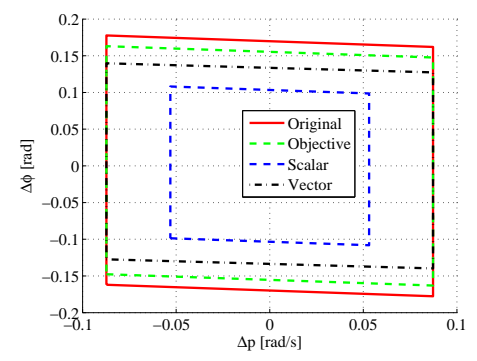

(c) $\Delta p-\Delta \phi$

Figure 9: Example of lateral $H=1$ recoverable sets scaled from an original recoverable set to an objective recoverable set. Note that both scalar and vector scaling produce decent approximations in this case. Also note that the vector scaling comes pretty close to replicating the objective set in some examples. 
Table 2: Examples of optimal scaling parameters $\lambda^{*}$

\begin{tabular}{clll}
\hline Motion & Original & Objective & $\lambda^{*}$ \\
\hline Longitudinal & 1 & 2 & 0.3210 \\
Longitudinal & 2 & 1 & 0.3695 \\
Lateral & 1 & 2 & 0.6087 \\
Lateral & 2 & 1 & 0.9688 \\
\hline
\end{tabular}

Table 3: Examples of optimal scaling parameters $\hat{\lambda}^{*}$

\begin{tabular}{clll}
\hline Motion & Original & Objective & $\hat{\lambda}^{*}$ \\
\hline Longitudinal & 1 & 2 & {$[0.6792,0.3346,1.000,0.8251,0.6390,0.5099,0.8209,0.8255]$} \\
Longitudinal & 2 & 1 & {$[0.6553,0.9570,1.000,0.5092,0.8470,0.8765,0.7584,0.7619]$} \\
Lateral & 1 & 2 & {$[0.7384,1.0000,1.0000,0.7861,0.6840,0.6567,0.7005,0.6660]$} \\
Lateral & 2 & 1 & {$[0.9803,1.0000,0.9851,0.9203,1.0639,1.0869,0.9692,0.9712]$} \\
\hline
\end{tabular}

\section{An Icing Example}

An icing example is now presented in which the effects of reduced lift and increased drag as consequences of icing are considered. ${ }^{20,21}$ This example will show how a $H=1$ recoverable set at one flight condition of $U_{0}=500 \mathrm{ft} / \mathrm{s}, h=1000 \mathrm{ft}$, and $\alpha=2 \mathrm{deg}$ can be scaled to estimate the $H=1$ recoverable set at another objective flight condition of $U_{0}=580 \mathrm{ft} / \mathrm{s}, h=1000 \mathrm{ft}$, and $\alpha=2 \mathrm{deg}$. These are the same flight conditions used in Section VI.

For this example, the coefficients of lift, $C_{L}$, and drag, $C_{D}$, are altered in order to reflect increased drag and decreased lift consistent with wing icing. This method is consistent with what is done in. ${ }^{20,21}$ To do this, each stability derivative that is a component of the lift force or the drag force and appears in the linear models is multiplied by a factor so that

$$
C_{L, \text { new }}=1 / 2 C_{L, \text { nom }}, \quad C_{D, \text { new }}=2 C_{D, \text { nom }} .
$$
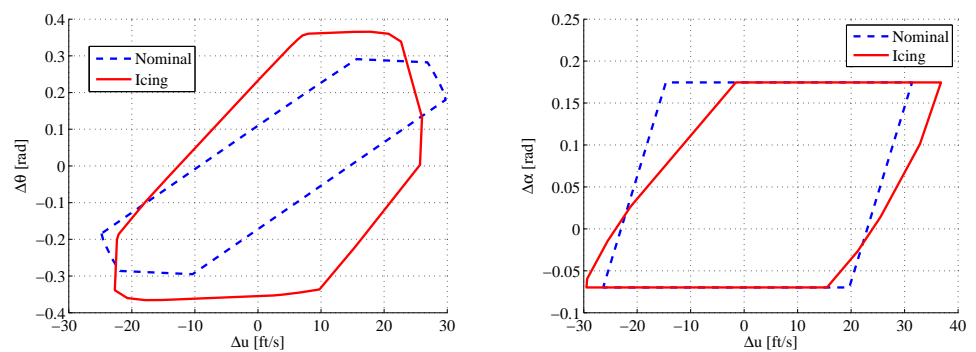

(a) $\mathrm{H}=1$ recoverable set changes due to icing in the $\Delta u-\Delta \theta$ plane.

(b) $\mathrm{H}=1$ recoverable set changes due to icing in the $\Delta u-\Delta \alpha$ plane.

Figure 10: Examples of how the icing affects the $\mathrm{H}=1$ recoverable sets in the $\Delta u-\Delta \theta$ plane(a) and the $\Delta u-\Delta \alpha$ plane(b). Note the icing is more predominately seen in (a) than in (b).

Figure 10 presents the changes in the $H=1$ recoverable set for the changed dynamics in the $\Delta u-\Delta \theta$ and $\Delta u-\Delta \alpha$ planes. The observed variation, particularly in the $\Delta u-\Delta \theta$ plane, is consistent with what physically 
occurs when an aircraft experiences an increase in drag and a decrease in lift. The aircraft must pitch more at lower deviations in nominal airspeed to avoid constraint violations and cannot fly as slowly as before without increased negative pitch.

Figure 11 provides the results of the scalings. The scalings that are used to perform these transformations are the scalings calculated for the nominal dynamics and found in Tables 2 and 3. Also note that with the performed transformation, the scaled recoverable sets are within the actual calculated recoverable sets thus these scaled sets are not in violation of any constraint. This suggests that this is a valid method of predicting safe sets at another flight condition when only knowledge of the current flight condition is available.

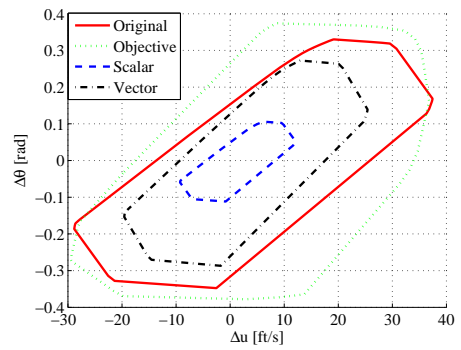

(a) $\Delta u-\Delta \theta$

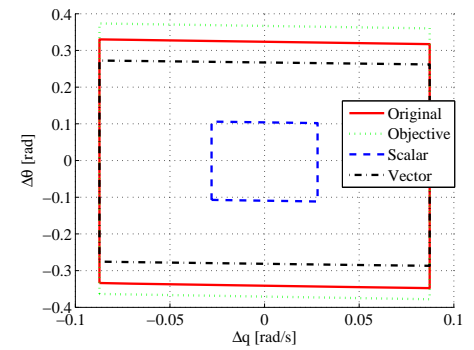

(b) $\Delta \alpha-\Delta \theta$

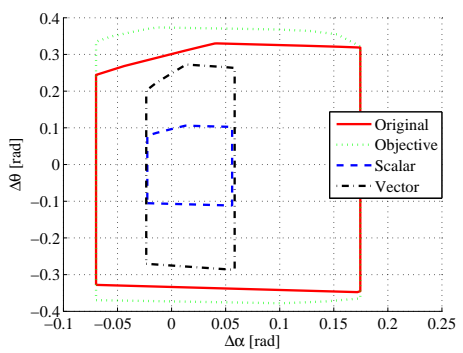

(c) $\Delta q-\Delta \theta$

Figure 11: Scaling of the icing scenario recoverable sets

\section{Nonlinear Recoverability}

Consider a nonlinear system,

$$
x(t+1)=f(x(t), v(t), \theta),
$$

with constraints given by

$$
G x(t) \leq F,
$$

where $\theta$ are model parameters. As in the linear case, we assume that $v(t)$ is given by

$$
v(t)=\bar{C} \bar{x}(t)+\rho,
$$

where $\bar{x}$ is generated by an auxiliary system,

$$
\bar{x}(t+1)=\bar{A} \bar{x}(t) .
$$

Let

$$
p=\left[\begin{array}{c}
\bar{x}(0) \\
\rho
\end{array}\right] .
$$

The state $x(0)$ of (35) is said to be recoverable if there exists $p$ such that the ensuing trajectory of (35) with $v(t)$ generated by (37) and (38) satisfies the constraints (36) for all $t \in Z^{+}$.

Because the underlying system model is nonlinear, finding $p$ that demonstrates that the state $x(0)$ is recoverable can be complicated. We seek approaches that exploit approximations and reduce the problem to a finite set of easier solvable problems.

Specifically, to check whether $x(0)$ is a recoverable state, we employ a procedure based on computing parametric sensitivities. Suppose that $P$ is the set in which the search for $p$ will be undertaken. Let

$$
p^{j} \in P, j=1, \cdots, N_{p}
$$

be a finite set of points in $P$. We can approximate the response of $(35),(37),(38)$ by a first order Taylor series expansion,

$$
x(t)=x^{j}(t)+\left.\frac{\partial x}{\partial p}\right|_{j}(t)\left(p-p^{j}\right)+\frac{\partial x}{\partial \theta}(t)\left(\theta-\theta^{0}\right)+\text { higher order terms, }
$$


where $x^{j}(t)$ denotes the response with $p=p^{j}$. We now discuss how (i) the approximation (41) can be used in the search for a feasible $p$ online and (ii) how the parametric sensitivities $\left.\frac{\partial x}{\partial p}\right|_{j}(t),\left.\frac{\partial x}{\partial \theta}\right|_{j}(t)$ can be computed off-line and stored for online use.

The check for feasible $p$ can be reduced to solving $N_{p}$ Linear Programming (LP) problems of the form,

$$
\begin{gathered}
\left\|p-p^{j}\right\|_{\infty} \rightarrow \min _{p}, \text { subject to } \\
G\left(x^{j}(t)+\left.\frac{\partial x}{\partial p}\right|_{j}\left(p-p^{j}\right)+\frac{\partial x}{\partial \theta}\left(\theta-\theta^{0}\right)\right) \leq F-\epsilon, t=0, \cdots, T,
\end{gathered}
$$

where $\epsilon>0$ is a constraint tightening parameter. If there exists a feasible solution to one of the $N_{p}$ problems, $p$, such that $\left\|p-p^{j}\right\| \leq \Delta$, where $\Delta$ is the chosen trust region size for the approximation (41) then $x(0)$ is recoverable.

To compute the parametric sensitivity matrix $\left.\frac{\partial x}{\partial p}\right|_{j}$, we first employ the solution to (37)

$$
v(t)=c(t) p, \quad c(t)=\left[\bar{C} \bar{A}^{t}, \quad I\right] .
$$

Then by taking partial derivatives in (35) and employing chain rule, we arrive at

$$
\left.\frac{\partial x}{\partial p}\right|_{j}(t+1)=\left.\left.\frac{\partial f}{\partial x}\right|_{x^{j}(t), v^{j}(t)} \frac{\partial x}{\partial p}\right|_{j}(t)+\left.\frac{\partial f}{\partial v}\right|_{x^{j}(t), v^{j}(t)} c(t) .
$$

This is the matrix difference equation with the initial condition defined by

$$
\left.\frac{\partial x}{\partial p}\right|_{j}(0)=0
$$

The trajectories $x^{j}, v^{j}$ are the simulated trajectories with the given $x(0)$ and with $p=p^{j}$ and $\theta=\theta^{0}$.

To demonstrate this method, we employ the longitudinal flight model from reference. ${ }^{5}$ In this model, the states are airspeed, $V$, and flight path angle, $\gamma$, and the controls are thrust, $T$, and angle of attack, $\alpha$.

$$
\begin{array}{r}
\dot{V}=-\frac{\rho S V^{2}}{2 m}\left(C_{D 0}+C_{D \alpha} \alpha+C_{D \alpha^{2}} \alpha^{2}\right)-g \sin (\gamma)+\frac{T}{m}, \\
\dot{\gamma}=-\frac{g}{V} \cos (\gamma)+\frac{\rho S V}{2 m}\left(C_{L 0}+C_{L \alpha} \alpha\right) .
\end{array}
$$

In this model $\rho=1.225 \mathrm{~kg} / \mathrm{m}^{3}, S=260 \mathrm{~m}^{2}, m=120,000 \mathrm{~kg}, C_{L 0}=1.0656, C_{L \alpha}=6.0723, C_{D 0}=0.1599$, $C_{D \alpha}=0.5035, C_{D \alpha^{2}}=2.1175$ are all constants. The thrust is limited to $20546 \leq T \leq 410920 \mathrm{~N}$ and the angle of attack is limited to $0 \leq \alpha \leq 14.5 \mathrm{deg}$.

For the results presented below, a trim condition is selected from the space $55 \leq V \leq 85 \mathrm{~m} / \mathrm{s}$ gridded with 20 points and $0 \leq \gamma \leq 15$ deg gridded with 15 points. Trajectories are simulated from this initial condition through the application of constant control values. These constant control values are the $\rho$ in (37). For convenience, the constant values selected are a subset of those that produce the trim conditions within the space described above. The trajectories are then constrained so that they do not deviate more than $\pm 10 \mathrm{~m} / \mathrm{s}$ or $\pm 5 \mathrm{deg}$ from the initial condition or the boundaries of the search space-which ever comes first. If the ensuing trajectories satisfy the constraints, the control inputs and final trim conditions are selected as possible options. If not, (42) is tested and if $p^{j}$ is found such that $\left\|p-p^{j}\right\| \leq \Delta$, then the control inputs and final conditions are also selected as possible options.

Figures 12a-12c demonstrate these results for the initial condition $x_{0}=[66.0526,6.67]^{T}$ with a $\Delta=$ 39037 -this value is $10 \%$ of the difference between the maximum and minimum thrust. Figure 12a shows all of the constant control inputs that were searched, Figure 12b gives the control input values that were selected, and Figure 12c shows the final trim conditions that the aircraft could reach without violating constraints. The search over the full set of possible constant control inputs took 1.35 seconds. We chose the search space to be much more sparse than the trim condition space to improve the speed of the calculations. We should note that in Figures 12b-12c, the red points, the ones that the simulated trajectory satisfies the set of constraints, produces values that are within the constraints described above. However, the blue points do deviate outside of these bounds because there exists $p^{j}$ such that the point is considered recoverable. The green points indicate the constant initial control input in Figure 12b and the initial trim condition in 12c.

In these results, we assumed $\theta=\theta^{0}$. However, this method can handle changes in flight parameters such as the coefficients of drag and lift assuming these changes are able to be identified online. 


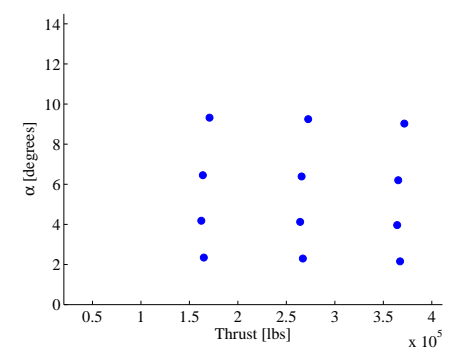

(a) Constant control input space

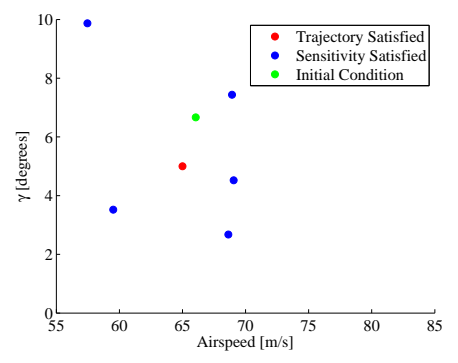

(b) Selected constant control inputs

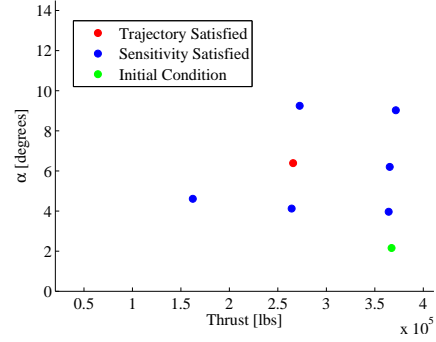

(c) Selected ensuing final trim conditions.

Figure 12: The constant control inputs or ensuing final trim conditions that do not violate constraints (red) and for which a suitable recovery sequence can be generated (blue)

\section{Conclusion}

This paper has proposed approaches to computing and predicting safe and recoverable sets of aircraft dynamics based on linearized discrete-time models and nonlinear models. These approaches have targeted an implementation for which real-time on-board computations are feasible. Illustrations and examples of constructing safe and recoverable sets have been given, including for a case study of aircraft icing. The recoverable sets can be used to facilitate aircraft flight planning-either automated or via a pilot advisory-in loss of control situations.

\section{Appendix A: Aircraft Model Examples}

This appendix presents the equations used to calculate the linear longitudinal and lateral models for a given flight condition. First the longitudinal equations.

$$
\begin{gathered}
\dot{\Delta u}=\frac{-p_{d} S}{m U_{0}}\left(2 C_{D 0}+C_{D u}\right) \Delta u+\frac{p_{d} S}{m}\left(C_{L 0}+C_{D \alpha}\right) \Delta \alpha+\frac{p_{d} S c}{2 m U_{0}} C_{D q} \Delta q-g \Delta \theta+\frac{p_{d} S}{m} C_{D \delta_{e}} \delta_{e}+\frac{T_{m a x}}{m} \delta_{t h} \\
\dot{\Delta \alpha}=\frac{-p_{d} S}{m U_{0}^{2}}\left(2 C_{L 0}+C_{L u}\right) \Delta u+\frac{-p_{d} S}{m U_{0}}\left(C_{L \alpha}+C_{D 0}\right) \Delta \alpha+\left(1-\frac{p_{d} S c}{2 m U_{0}^{2}} C_{L q}\right) \Delta q-\frac{p_{d} S}{m U_{0}} C_{L \delta_{e}} \delta_{e} \\
\dot{\Delta q}=\frac{p_{d} S C}{I_{y y} U_{0}}\left(2 C_{m 0}+C_{m u}\right) \Delta u+\frac{p_{d} S c}{I_{y y}} C_{m \alpha} \Delta \alpha+\frac{p_{d} S c^{2}}{2 I_{y y} U_{0}} C_{m q} \Delta q-\frac{p_{d} S c}{I_{y y}} C_{L \delta_{e}} \delta_{e} \\
\dot{\Delta \theta}=\Delta q
\end{gathered}
$$

Now the lateral equations.

$$
\begin{gathered}
\dot{\Delta \beta}=\frac{-p_{d} S}{m U_{0}}\left(C_{D 0}+C_{E \beta}\right) \Delta \beta+\frac{-p_{d} S b}{2 m U_{0}^{2}} C_{E p} \Delta p+\left(\frac{p_{d} S b}{2 m U_{0}^{2}} C_{E r}-1\right) \Delta r-\frac{p_{d} S}{m U 0} C_{E \delta a} \delta a-\frac{p_{d} S}{m U 0} C_{E \delta r} \delta r \\
\dot{\Delta p}-\frac{I_{x z}}{I_{x x}} \dot{\Delta r}=\frac{p_{d} S b}{I_{x x}} C_{l \beta} \Delta \beta+\frac{p_{d} S b^{2}}{2 I_{x x} U_{0}} C_{l p} \Delta p+\frac{p_{d} S b^{2}}{2 I_{x x} U_{0}} C_{l r} \Delta r \frac{p_{d} S b}{I_{x x}} C_{l \delta a} \delta a+\frac{p_{d} S b}{I x x} C_{l \delta r} \delta r \\
\dot{\Delta r}-\frac{I_{x z}}{I_{z z}} \dot{\Delta p}=\frac{p_{d} S b}{I_{z z}} C_{n \beta} \Delta \beta+\frac{p_{d} S b^{2}}{2 I_{z z} U_{0}} C_{n p} \Delta p+\frac{p_{d} S b^{2}}{2 I_{z z} U_{0}} C_{n r} \Delta r \frac{p_{d} S b}{I_{z z}} C_{n \delta a} \delta a+\frac{p_{d} S b}{I z z} C_{n \delta r} \delta r \\
\dot{\Delta \phi}=\Delta p
\end{gathered}
$$

In these equations, the dynamic pressure is $p_{d}=\frac{1}{2} \rho U_{0}^{2}, C_{X X}$ are the supplied stability derivatives and $I_{X X}$ is the moment of inertia about a particular axis. 


\section{Acknowledgments}

This work was supported in part by the National Aeronautics and Space Administration under Cooperative Agreement NNX12AM54A. The authors would also like to thank Professor Dennis Bernstein in his help with the equations in Appendix A as well as graduate student researchers Ming-Jui Yu and Sweewarman Balachandran for their help and support on the project.

\section{References}

\footnotetext{
${ }^{1}$ Lombaerts, Thomas, Quping Chu, Jan Albert Mulder, and Diederick Joosten. "Flight control reconfiguration based on a modular approach." In Fault Detection, Supervision and Safety of Technical Processes, pp. 259-264. 2009.

${ }^{2}$ Lombaerts, Thomas, Eddy Van Oort, Q. P. Chu, J. A. Mulder, and Diederick Joosten. "Online Aerodynamic Model Structure Selection and Parameter Estimation for Fault Tolerant Control." Journal of guidance, control, and dynamics 33, no. 3 (2010): 707-723.

${ }^{3}$ Lombaerts, Thomas, Ping Chu, and Jan Albert Bob Mulder. "Flight control reconfiguration based on online physical model identification and nonlinear dynamic inversion." In Fault tolerant flight control, pp. 363-397. Springer Berlin Heidelberg, 2010.

${ }^{4}$ Lombaerts, T. J. J., Chu, Q. P., Mulder, J. A., Joosten, D. A. (2011). Modular flight control reconfiguration design and simulation. Control Engineering Practice, 19(6), 540-554.

${ }^{5}$ Lombaerts, T. J., Schuet, S. R., Wheeler, K. R., Acosta, D. M., and Kaneshige, J. T., "Safe maneuvering envelope estimation based on a physical approach," AIAA Guidance, Navigation, and Control Conference, 18-22 August, 2013, Boston, Massachusetts.

${ }^{6}$ Bayen, A. M., Mitchell, I. M., Osihi, M. K., Tomlin, C. J. (2007). Aircraft autolander safety analysis through optimal control-based reach set computation. Journal of guidance, control, and dynamics, 30(1), 68-77.

${ }^{7}$ Barlow, J., Stepanyan, V., Kalmanhe, K. Estimating Loss-of-Control: A Data-Based Predictive Control Approach. AIAA Guidance, Navigation, and Control Conference, 08-11 August 2011, Portland, Oregon.

${ }^{8}$ Stepanyan, V., Krishnakumar, K., Barlow, J., Bijl, H. (2011). Adaptive Estimation Based Loss of Control Detection and Mitigation. AIAA Guidance, Navigation, and Control Conference, 08-11 August 2011, Portland, Oregon.

${ }^{9}$ E.G. Gilbert and K.-T. Tan, Linear systems with state and control constraints: The theory and application of maximum output admissible sets, IEEE Transactions on Automatic Control, Volume 36, No. 9, 1991.

${ }^{10}$ John Lygeros, On reachability and minimum cost optimal control, Automatica, Volume 40, Issue 6, June 2004, Pages 917-927, ISSN 0005-1098, 10.1016/j.automatica.2004.01.012.

${ }^{11}$ H. G. Kwatny, J-E T. Dongmo, B-C Chang, G. Bajpai, M. Yasar, and C. Belcastro, Aircraft Accident Prevention: Lossof-Control Analysis, Proceedings AIAA Guidance Navigation and Control Conference and Exhibit, Chicago, 10-13 August, 2009.

${ }^{12}$ H. G. Kwatny, J-E. T. Dongmo, R. C. Allen, B-C Chang, G. Bajpai, Loss-of-Control: Perspectives on Flight Dynamics and Control of Impaired Aircraft, AIAA Conference on Guidance, Navigation and Control, Toronto, Ontario, Canada, August $2-5,2010$.

${ }^{13}$ R. C. Allen and H. G. Kwatny, Manueverability and Envelope Protection in the Prevention of Aircraft Loss of Control, 8th Asian Control Conference (ASCC 2011), Kaohsiung, Taiwan, May 15-18, 2011.

${ }^{14}$ H. G. Kwatny and R. C. Allen, "Safe Set Maneuverability of Impaired Aircraft," Proceedings AIAA Guidance Navigation and Control Conference and Exhibit, Chicago, 12-16 August, 2012.

${ }^{15}$ Kwatny, H. G., Dongmo, J. E. T., Chang, B. C., Bajpai, G., Yasar, M., Belcastro, C. (2012). Nonlinear Analysis of Aircraft Loss of Control. Journal of Guidance, Control, and Dynamics, 36(1), 149-162.

${ }^{16}$ Nguyen, N., Trinh, K., Frost, S., Reynolds, K., "Couple Aeroelastic Vortex Lattice Modeling of Flexible Aircraft," 29th AIAA Applied Aerodynamics Conference, Honolulu, Hawaii, 27-30 June, 2011

${ }^{17}$ L. Tang, M. Roemer, J. Ge, A. Crassidis, J. Prasad, C. Belcastro, "Methodologies for Adaptive Flight Envelope Estimation and Protection," Proceedings AIAA Guidance Navigation and Control Conference, Chicago, Illinois, 10-13 August, 2009

${ }^{18}$ Menon, P. K., Sengupta, P., Vaddi, S., Yang, B. J., Kwan, J. (2013). Impaired Aircraft Performance Envelope Estimation. Journal of Aircraft, 1-15.

${ }^{19}$ J. Wilborn, J. Foster, "Defining Commercial Transport Loss-of-Control: A Quantitative Approach," AIAA Atmospheric Flight Mechanics Conference and Exhibit, Providence, Rhode Island, 16-19 August 2004.

${ }^{20}$ Bragg, M. B., Basar, T., Perkins, W. R., Selig, M. S., Voulgaris, P. G., Melody, J. W., Sarter, N. B. (2002). Smart icing systems for aircraft icing safety. AIAA Paper, 813.

${ }^{21}$ Reehorst, Andrew L., Harold E. Addy Jr, and Renato O. Colantonio. "Examination of Icing Induced Loss of Control and Its Mitigations." AIAA Paper 8140 (2010).

${ }^{22}$ Urnes, J. M., Reichenbach Sr, E. Y., Smith, T. A. (2008, August). Dynamic Flight Envelope Assessment and Prediction. In AIAA Guidance Navigation and Control Conference (pp. 18-21).

${ }^{23}$ Pandita, R., Chakraborty, A., Seiler, P., Balas, G. (2009). Reachability and region of attraction analysis applied to gtm dynamic flight envelope assessment. In AIAA Guidance, Navigation, and Control Conference, number AIAA-2009-6258.

${ }^{24}$ van Oort, E. R., Chu, Q. P., Mulder, J. A. (2011). Maneuver Envelope Determination through Reachability Analysis. In Advances in Aerospace Guidance, Navigation and Control (pp. 91-102). Springer Berlin Heidelberg.

${ }^{25}$ C. M. Belcastro and J. V. Foster, "Aircraft Loss-of-Control Accident Analysis," in Proc. AIAA Guidance, Navigation, and Control Conference, Toronto, Ontario, 2010.
} 
${ }^{26}$ NTSB, "Loss of Thrust in Both Engines After Encountering a Flock of Birds and Subsequent Ditching on the Hudson River," [Online]. Available: http://www.ntsb.gov/doclib/reports/2010/aar1003.pdf. [Accessed 17 April 2012].

${ }^{27}$ Brian L. Stevens and Frank L. Lewis, 2003."Aircraft Control and Simulation". John Wiley and Sons. Hoboken, NJ.

${ }^{28}$ B.Pluymers, J.A. Rossiter, J.A.K. Suykens, B. De Moor, 2005. "The Efficient Computation of Polyhedral Invariant Sets for Linear Systems with Polytopic Uncertainty". In Proceedings of 2005 American Control Conference pp. 804-809. Portland, OR, June.

${ }^{29}$ D.Q. Maynem, M.M. Seron, S.V. Rakovic, 2005. "Robust model predictive control of constrained linear systems with bounded disturbances". Automatica, vol. 41, pp. 219-224. January, 2005.

${ }^{30}$ Avishai Weiss, Moorgan Baldwin, R. Scott Erwin, Ilya Kolmanovsky, 2012. "Spacecraft Constrained Maneuver Planning Using Positively Invariant Constraint Admissible Sets". In Proceedings of Itzhack Y. Bar-Itzhack Memorial Symposium on Estimation, Navigation, and Spacecraft Control, pp. 459-474. Haifa, Israel. October, 2012.

${ }^{31}$ Ilya Kolmanovsky, E.G. Gilbert, H.E. Tseng, 2009. "Constrained Control of Vehicle Steering". In Proceedings 2009 IEEE Multi-conference on Systems and Control, pp 576-581. Saint Petersburg, Russia. July, 2009.

${ }^{32}$ E.G. Gilbert, C.J. Ong. "Linear Systems with Hard Constraints and Variable Set Points: Their Robustly Invariant Sets".

${ }^{33}$ Kalabic,U.; Kolmanovsky,I.; Buckland, J.; Gilbert, E., "Reference and extended command governors for control of turbocharged gasoline engines based on linear models," 2011 IEEE International Conference on Control Applications, pp. 319-325, 2011

${ }^{34}$ I Kolmanovsky, E.G. Gilbert. "Maximal output admissible sets for discrete-time systems with disturbance inputs". In Proceedings 1995 American Conference on Control, pp 1995-1999, 1995

${ }^{35}$ I Kolmanovsky, E.G. Gilbert. "Theory and computations of disturbance invariant sets for discrete-time linear systems". Mathematical Problems in Engineering, Volume 4, pp. 317-367, 1998.

${ }^{36}$ Nikolaos Athanasopoulos, George Bitsoris. "Invariant set computation for constrained uncertain dsicrete-time linear systems". In Proceedings IEEE 2010 Conference on Decisions and Control, pp 5227-5232, December, 2010.

${ }^{37}$ D.Q. Mayne, J.B. Rawlings, C.V. Rao, P.O.M Scokaert. "Constrained model predictive control: Stability and optimality". Automatica vol 36, pp 789-814, 2000.

${ }^{38}$ F. Blanchini and S. Miani, 2008. "Set-Theoretic Methods in Control". Birkhauser. Boston, MA. 